\title{
Study on signs of defects in the image of the surface of flat- rolled products
}

\author{
Oksana Logunova ${ }^{1}$, Palvan Kalandarov ${ }^{2}$, Evgeny Garbar $^{1 *}$, Makhmud Abdullaev $^{3}$, Nodira Alimova ${ }^{3}$, Oybek \\ Matchanov $^{2}$, and Khusniddin Abdullaev ${ }^{2}$ \\ ${ }^{1}$ Magnitogorsk State Technical University named after G. I. Nosov, 455000 Magnitogorsk, Russia \\ ${ }^{2}$ Tashkent Institute of Irrigation and Agricultural Mechanization Engineers, 100000 Tashkent, Uzbekistan \\ ${ }^{3}$ Tashkent State Technical University, 100095 Tashkent, Uzbekistan
}

\begin{abstract}
Currently, more and more challenges of modern industrial enterprises require an increase in the reliability of the information on the quality of products. This becomes possible when using digital technologies to assess the quality of products. The cited publication discusses the technology for recognizing defects in the surface of sheet products in images obtained from cameras of the strip inspection system during rolling. The authors proposed a classification of the signs of defects in the image and highlighted the most significant of them also suggested using geometric, optical and spectral features for images of flat-rolled products containing defects of different classes. The research results at this stage, obtained during the processing of digital images, showed that to identify a defect and reduce false-positive and false-negative alarms of the automated defect identification system, it is required to conduct a study of interval estimates and make decision-making rules based on intersection and merging of intervals; introduce additional classes that allow the introduction of signs that characterize the irregularity of the shape of defects and the characteristic location; the use of new technologies of soft computing will reveal the hidden patterns of the manifestation of defects in the images of the surface of the steel strip.
\end{abstract}

\section{Introduction}

Cold-rolled steel with subsequent annealing and galvanized coating is used in industry for the manufacture of car bodies, housings for household appliances and the construction industry. The quality of the metal surface is one of the key criteria for the consumers [1]. When assessing the surface quality, both organoleptic methods and automated systems for recognizing surface defects (ASRSD) are used that analyze images of the rolled surface obtained at the sections of the , such as a continuous galvanizing aggregate(ACG) and a continuous hot-dip galvanizing aggregate(AHG) of PJSC Magnitogorsk Iron and Steel Works "(PJSC" MMK ") ASRDP, used in production, structurally consist of sensors, network interfaces, a defect detection unit, a database [2] (Fig. 1).

The sensors consist of several cameras, physically combined into a single unit, installed at the top and bottom of the strip. The existing ASRSD has a sufficiently high accuracy in detecting defects, however they have drawbacks too. The main disadvantages include false positive and false negative positives and incorrect determination of the defects. These disadvantages lead to an increase in production costs, so, for example, a large number of false positives enlarge the number of labor who needs to inspect each section and decide on the presence of defects. False negative alarms lead to the fact that products with a defect are sent to the consumer, who forms a complaint. Furthermore, a false determination of the class of defect does not allow making a timely corrective action on the unit or on previous production redistributions. It is therefore essential for eliminating the shortcomings to develop a more accurate method for assessing the quality of the strip surface. Modern methods of image pattern recognition include several stages, such as removing noise [3], determining the region of potential defect location, highlighting the defect area, determining the values of image features, followed by using neural networks to determine the defect class. The features of objects in the image are considered in [4-12]. In [8], the features of images are considered for further use of features in the classification of a defect.

"Corresponding author: evgenyiam@yandex.ru 


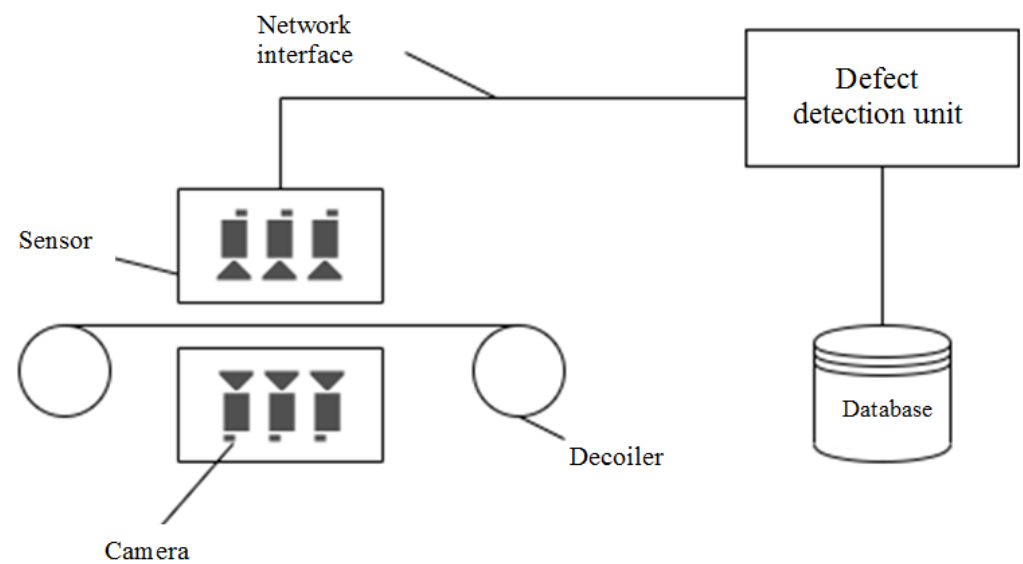

Fig. 1. Structure of automated systems for recognizing surface defects

\section{Research methods}

\subsection{Classification of signs of defects on the surface of steel plate}

The result of the work of the ASRSD is a set of digital images that are subjected to automated processing. The digital image supplied to the input of the defect detection unit is set by the values of the pixel brightness. To identify and classify defects on the surface of a metal sheet, it is necessary to enter features. The experts in the field proposed a classification of signs of defects by signs: geometric parameters, optical signs by brightness and spectral signs by the presence of texture Fig. 2.

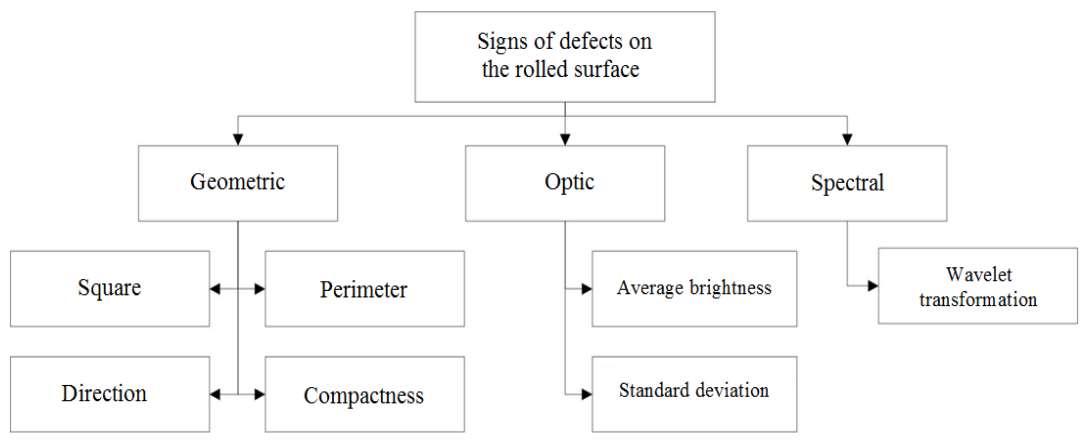

Fig. 2. Classification of signs of defects on the surface of steel sheet

For each feature, quantitative characteristics have been introduced, which make it possible to identify the presence of a defect on the surface in automatic mode.

\subsection{Quantitative characteristics of signs of defects}

Table 1. Formulas for quantifying geometric features

\begin{tabular}{|c|c|c|}
\hline Description & Designation & Formula \\
\hline $\begin{array}{l}\text { Defect area - the number of points on the binarized image that belong to } \\
\text { the defect zone }\end{array}$ & $S$ & $S=\sum_{(x, y) \in R} 1$ \\
\hline Perimeter - the number of points on the border of the defect area & $P$ & $P=\sum^{1} 1$. \\
\hline $\begin{array}{l}\text { Compactness - dimensionless quantity invariant to changes in scale and } \\
\text { rotation }\end{array}$ & $q$ & $\begin{array}{l}(x, y) \in G_{R} \\
q=\frac{P^{2}}{S}\end{array}$ \\
\hline $\begin{array}{l}\text { The direction of the defect [9] relative to the movement of the strip - the } \\
\text { angle between the line drawn in the middle of the smaller circumscribed } \\
\text { circumscribed rectangle and the direction of movement of the strip }\end{array}$ & $\alpha$ & $\begin{array}{l}\text { An example of determining } \\
\text { the angle is shown in Fig- } \\
\text { ure } 3 \text {. }\end{array}$ \\
\hline
\end{tabular}

Notice: The table contains designations: $\mathrm{R}-$ defect area; $\mathrm{G}_{\mathrm{R}}-$ defect boundary 
For the class of geometric features, formulas are introduced (see Table 1.)

The value of compactness takes on a minimum value for a shape close to a circle, which corresponds to such defects as holes and technological apertures when marking the welding spots of coils.

\section{Defect direction}

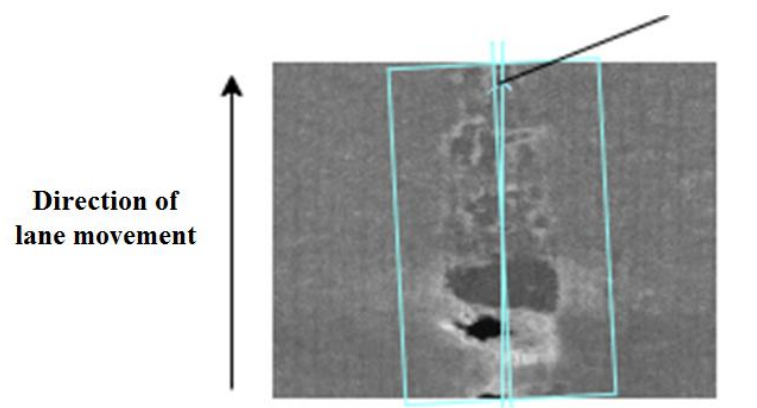

Fig. 3. Scheme for determining the direction of a defect on the rolled surface

For the class of optical features, a formula is used to calculate the average image brightness and the standard deviation from the average brightness.

Average value of brightness of defect pixels:

$$
b=\frac{1}{S} \sum_{(x, y) \in R} I(x, y) ;
$$

here $I(x, y)$ - two-dimensional matrix of brightness values of the image received from the ASRSD

The standard deviation of the brightness of the image obtained from ASRSD:

$$
\sigma=\sqrt{\frac{1}{S} \sum_{(x, y) \in R}(b-I(x, y))^{2}} .
$$

For spectral features, the formulas were used for calculating the wavelet transform coefficients given in [10]:

$$
b w_{z}^{j}=\frac{1}{n_{j}} \sum_{(x, y) \in R} w_{z}^{i}(x, y),
$$

When $i$-wavelet transform level 1,2; $\mathrm{z}$ - conversion direction is horizontal, vertical and diagonal; $w_{z}^{j}$ - wavelet transform coefficient at level I in direction $\mathrm{z} ; n_{j}$ - the number of points of the defect area at the - $\mathrm{m}$ level of the wavelet transform.

\section{Results and Discussions}

Information gained from accumulated in the conditions of operation in the conditions of the sheet-rolling shop of PJSC "MMK" made it possible to calculate the signs of defects. As a test example, images of surface defects of different classes were selected: scale (Fig. 4a), transverse cracks (Fig. 4b), and annealing tear (Fig. 4c).
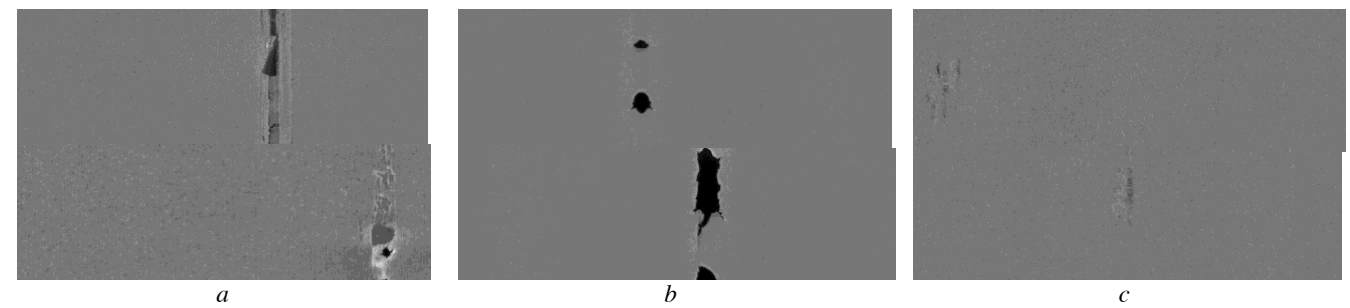

Fig. 4. Image of the "scale" (a), "transverse cracks" (b) and "annealing tear" (c) defects on the surface of the steel sheet

The quantitative calculation process was carried out using a software product. The calculation results are shown in Table 2 . 
The results obtained allow asserting the presence of interval estimates of the image for each feature and each type of defects. These estimates can be used to construct complex rules for making a decision on the presence and type of defect on the surface of flat-rolling product.

In the state policy of modern states, the red line is the challenge of introducing and developing digital technologies on production platforms. The level of development of computer technology, software and fundamental research in the field of image processing allow these technologies to be adapted to heavy industry and, in particular, to ferrous metallurgy. The present investigation in this area [13-16] has once again shown that the fundamental algorithms for processing digital images, on the one hand, are of the same type, but on the other hand, they require refinement and adaptation to the conditions of the problems being solved. Under the conditions of the above study, it is required to expand the classification features and develop decision-making techniques based on interval estimates. It is to the issues of that further research on the above topics will be devoted.

Table 2. Feature values for different classes of defects

\begin{tabular}{lccc}
\hline \multirow{2}{*}{ Defect symptom } & \multicolumn{3}{c}{ Defects } \\
\cline { 2 - 4 } & Scale & Transverse cracks & Annealing tear \\
\hline Square & $644 \ldots 6288$ & $139 \ldots 1735$ & $99 \ldots 1679$ \\
Perimeter & $144 \ldots 1819$ & $94 \ldots 308$ & $72 \ldots 378$ \\
Compactness & $146 \ldots 526$ & $23 \ldots 69$ & $55 \ldots 127$ \\
Direction & $-5 \ldots+5$ & $78 \ldots 93$ & $15 \ldots 90$ \\
Average brightness value & $62 \ldots 101$ & $24 \ldots 48$ & $21 \ldots 100$ \\
Root mean square deviation & $18 \ldots 23$ & $15 \ldots 29$ & $11 \ldots 22$ \\
$1^{\text {st }}$ order wavelet coefficients & $31 \ldots 50$ & $13 \ldots 29$ & $7 \ldots 32$ \\
$2^{\text {nd }}$ order wavelet coefficients & $15 \ldots 24$ & $6 \ldots 13$ & $15 \ldots 69$ \\
\hline
\end{tabular}

\section{Conclusions}

a) To determine the presence of a defect on the surface of a steel strip, it is proposed to use three classification criteria at the first level: geometric, optical and spectral.

b) For each class, quantitative measures have been introduced, which are necessary to identify the fact of the presence of a defect on the surface.

c) For a test sample of images, all quantitative parameters of each class were calculated using a software product.

d) The calculation results made it possible to obtain an interval estimate that can be used to identify a defect and reduce false-positive and false-negative alarms of the ASRSD.

e) To increase the accuracy of determining the class and type of defect, it is required:

- $\quad$ carry out a study of interval estimates and make decision rules based on the intersection and merging of intervals;

- to introduce additional classes that allow the introduction of signs that characterize the irregularity of the forms of defects and the characteristic location;

f) The use of new technologies of soft computing reveals the hidden patterns of the manifestation of defects in the images of the surface of the steel strip.

\section{References}

1. E.A. Garbar, Digitalization in assessing the quality of the surface of sheet metal, Mathematical and Software Systems in Industrial and Social Spheres 2, 30-34 (2019)

2. M.I. Kuzmin, Information-measuring system for detecting surface defects in sheet metal based on the method of neighborhoods, Candidate of Sciences Dissertation, Orenburg State University, Orenburg (2016)

3. E.A. Garbar, Information processing in the express assessment of the presence of a surface defect in galvanized steel, Mathematical and Software Systems in Industrial and Social Spheres 8, 23-30 (2020)

4. Yu.V. Puchkova, Formation of image recognition features based on stochastic geometry, Models, systems, networks in economics, technology, nature and society 2, 14 (2016)

5. A.Yu. Mikov, Mathematical support and structure of the intellectual support system of the control system for assessing the surface quality of cold-rolled rolled products, Electrical systems and complexes 1(30), 45-51 (2016)

6. K.M. Grishanov, Methods of Feature Extraction for Character Recognition, Science, Technology and Education 1(5), 110-119 (2016)

7. R.M. Haralick, Textural Features for Image Classification, IEEE Transactions on Systems, Man, and Cybernetic 6, 610-621 (1973)

8. I.S. Vasendina, Development of a method for pattern recognition in an image based on a structural approach Bulletin of the Bryansk State Technical University 1(54), 171-177 (2017) 
9. N.A. Maksimov, Analysis of the shape of images and recognition of objects based on skeletal-contour representation, Scientific Bulletin of the Moscow State Technical University of Civil Aviation 207, 67-75 (2014)

10. B.Zh. Zharlykasov, Methods for the extraction of image features for the purpose of searching and recognizing faces, New Information Technologies in Education and Science 1, 114-117 (2018)

11. P.I. Kalandarov, B.P. Iskandarov, Physicochemical measurements: measurement of the moisture content of brown coal from the Angrensk deposit and problems of metrological assurance, Measurement Techniques 55(7), 845-848 (2012)

12. P.I. Kalandarov, Z.M. Mukimov, O.S. Logunova, Design of grain humidity control devices for milles, Technical Science and Innovation 2, 227-233 (2021)

13. I.I. Matsko, Y.V. Snegirev, O.S. Logunova, Data acquisition and preparation methods for continuously cast billets quality analysis software, Applied Mechanics and Materials 110-116, 3557-3562 (2012)

14. O.S. Logunova, D.K. Devyatov, K.K. Nurov, Computerized quality estimates of continuous-cast billet, Steel in Translation 35(9), 36-42 (2005)

15. M.Yu. Narkevich, O.S. Logunova, V.D. Kornienko, A.A. Nikolaev, A.N. Tyulyumov, N.V. Zlydarev, D.I. Deryabin, The quality of materials, products and structures in industrial safety: an empirical basis, Bulletin of the Magnitogorsk State Technical University 19, 90-101 (2021)

16. B.P. Iskandarov, P.I. Kalandarov, An analysis of the effect of interfering factors on the results of measurements of the moisture content of a material at high frequencies, Measurement Techniques 56, 827-830 (2013) 